Article

\title{
Innovation Diffusion of Mobile Applications in Social Networks: A Multi-Agent System
}

\author{
Lixin Zhou ${ }^{1}$, Jie Lin ${ }^{1}$, Yanfeng $\mathrm{Li}^{1}$ and Zhenyu Zhang ${ }^{1,2, *(1)}$ \\ 1 School of Economics and Management, Tongii University, Shanghai 200092, China; \\ zhoulixin1861@hotmail.com (L.Z.); linjie@tongji.edu.cn (J.L.); lyf19941128@126.com (Y.L.) \\ 2 Shanghai Municipal Engineering Design Institute (Group) Co., Ltd., Shanghai 200092, China \\ * Correspondence: zhangzhenyu7@foxmail.com
}

Received: 6 March 2020; Accepted: 1 April 2020; Published: 4 April 2020

check for updates

\begin{abstract}
Mobile application innovation diffusion can be used to enhance the reputation and competitiveness of Internet enterprises. However, few works have explored the process of mobile application innovation diffusion from the individual perspective. Therefore, this paper employs multi-agent methods to simulate the innovation diffusion of mobile applications from the perspective of social networks. Specifically, we combine psychology, sociology, game theory and network effect theory to model user behaviors of adoption and rejection decisions for mobile applications. The multi-agent model was built in Anylogic 8 to simulate the communication and interaction between individual users. Then, this paper discusses the evolution of decision-making of social network user groups with different network structures and network effects. We also investigate the impact of different firms' promotion on innovation diffusion. Our findings suggest firms could make better strategies and achieve better diffusion effects from mobile applications.
\end{abstract}

Keywords: mobile applications; innovation diffusion; multi-agent; social networks

\section{Introduction}

Nowadays, market competition in mobile applications is increasingly fierce. The success of the mobile application in market promotion not only helps Internet enterprises to seize the market and create business value, but also allows firms establish a good reputation and gain a competitive advantage [1-3].

The innovation diffusion of mobile applications always occurs in user groups. Since users can communicate and interact with others, most of the diffusion of mobile applications is based on each user in a social network [4,5]. Numerous studies indicate that the development of mobile internet and technology social networks, significantly reducing the cost of communication between users, makes communication and interaction very convenient [6,7]. As various mobile applications allow users to share happiness and dissatisfaction with others on the Internet, users' decisions on mobile applications are also influenced by social relations and social network structure [8,9]. In addition, research evidence also supports the fact that there are network effects in the diffusion of mobile applications; that is, the utility of mobile applications to users will increase with an increase in the number of applications [10]. Specifically, network effects can be divided into global and local network effects. Global network effect means that, when more users adopt innovative technology in the global environment, the value of mobile applications to users will be higher. While the local network effect refers to the effect of related users on the utility value, which emphasizing the interaction between users. The decision-making of users will largely depend on the decision-making of their surroundings. As the number of users adopting mobile applications increases, their neighbors also would like to adopt the same applications. Then, the value and utility of the mobile applications to the users will also increase, which ultimately 
affects user decision-making. In addition, the density of the network structure will affect the network effect. When the intensity of the network effects of the mobile applications is stronger, the value of the network effects will be greater. On the contrary, if the network effect is less intense, the user's decision will have less impact on the surrounding users [11-13].

In short, the diffusion of mobile applications in user groups is complex, which is influenced by the structure of the user's social network, the network effect and the interactive communication between each user. However, most researches have not yet answered the following important questions: First, how does a user's personal decision affect mobile application diffusion in the user group? Secondly, what are the effects of social network topology, network effect, enterprise promotion and other factors affect the decision-making of user group? These issues are necessarily addressed to promote the innovation diffusion of mobile applications.

Therefore, the present study seeks to explore the innovation diffusion of mobile applications from individuals to user groups. Since agent-based models [14,15] allow us to explore user behavior at the micro-level, we build a simulation model to describe individual decision-making and complex interaction between users of mobile applications in social networks. More specifically, we (i) analyze the user behavior characteristics of mobile applications, extracting the key factors that affect user decision-making. We then establish a conceptual model of user adoption and rejection decision-making, using a social learning method to present the process of user decision-making and interaction in social networks; (ii) conduct multi-agent models of mobile application innovation diffusion under different network structures and network effects; and (iii) investigate the impact of firm promotion on diffusion through simulation experiments, which will help enterprises make suitable strategies.

We make two key contributions to the literature. Firstly, our research offers a basic multi-agent model, which combines psychology theory, evolutionary game theory and social network analysis to simulate the diffusion of mobile applications from individual decisions to group behaviors. Secondly, our findings consider the impact of the user's personality and the neighbor's influence on personal decisions, providing firms promotion strategies and suggestions for the diffusion of mobile applications.

The remainder of the paper is organized as follows. First, we outline the literature on mobile applications, innovation diffusion and social networks. Secondly, we detail the design concept of our simulation model. Section 3 then implements the model based on the multi-agent method, which simulates mobile application innovation diffusion from social networks. The results of the simulation experiments are introduced and analyzed in Section 4. Finally, we conclude and provide suggestions for the marketing decision-making and management policy of enterprises.

\section{Related Work}

Recent research has explored the diffusion of new products in the market based on the Bass model $[16,17]$. Although the classical model can reflect the overall marketing activity and word of mouth (WOM) among consumers, the process of individual diffusion is still hard to understand $[14,18]$. Therefore, in order to describe the diffusion process from the micro level, this paper employed agent-based models to simulate the individual behavior (i.e., communication and interaction) in user groups, and accumulated the individual behaviors to obtain the diffusion results of the user groups.

\subsection{Agent-Based Modeling and Game Theory}

Agent-based modeling has become another typical method to study innovation diffusion in the past decade [19-21]. The simulation model abstracts the individual in the real-world into agents, and sets the attributes, characteristics and behavior rules of the agents, to simulate the dynamic interaction between different individuals. Since agent-based modeling enables fine-grained modeling of the interactions in social networks, this simulation model has been adopted to study the process of innovation diffusion [5,22,23]. Drawing on agent-based modeling, Ma and Nakamori developed a micro-diffusion model to explore the process of technological innovation from the perspective of each consumer and producer. In this model, producers can communicate with producers and 
develop the product to better meet consumers' preferences. This research reported on the influence of consumer interaction on innovation diffusion among the product design process [24]. Then, Stummer and Kiesling constructed an agent model to further simulate repetitive buying decisions under a multi-product competition market from time and space dimensions. The researchers demonstrated how the simulation model can be used to evaluate product launch strategies in a competitive setting, and studied the impact of production and sales policies on the diffusion of new products [25]. Although agent-based models allow us to represent rich behavior at the micro level, these models do not involve complex agent interactions, such as strategy learning, decision imitation and different individuals.

Game theory is a mathematically formalized strategic interaction theory; that is, the author not only pays attention to the outcome of the selected scheme, but also considers the possible outcome of other schemes in the decision-making process, which simply analyzes the gains and losses of users affected by other users' decisions [26]. It has been commonly used in marketing, in order to model competition between companies or customers [27]. Ozkane et al. employed game theory to simulate individual behavior in the process of the generation and evolution of innovation. This research provided a theoretical framework from which to figure out what users will do, to choose the best strategy based on other's decisions [28]. In addition, Zhang and Sun constructed an agent-based model to simulate users' innovative decisions from the perspective of game theory. The models are used to describe the evolution path and evolutionarily stable strategy of innovative behavior-based decisions, obtaining a sustainable competitive advantage for high-tech firms in a competitive environment [29]. Drawing on game theory, users can find better strategies by learning, constantly adjusting and improving innovation behaviors. In addition, game theory focuses on the interaction among different individual and user groups, so that users can explain the varying degrees of complexity of multi-agent models with differing strategic decisions [30]. Hereby, we adopt the game theory method to describe the multi-agent interaction of user diffusion behavior for mobile applications.

\subsection{Social Network Structure and Social Influence}

Prior studies on agent-based models have found that user relationship structure, such as the number of connections of an agent, the weighting of the connections, and so on, can also affect the result of innovation diffusion [31-33]. Numerous studies indicate that social network analysis is a common tool to describe realistic consumer networks. It can effectively describe the diffusion environment of user interaction [4,34]. Prior studies constructed the consumers' relationship network based on agent-based modeling and social network analysis, helping firms predict product proliferation in different networks $[35,36]$. Then, Choi and Byungtae pointed out that the external network perceived by the individual should be the local network itself. They investigated the proliferation of malicious mobile phone software in the network and considered enhanced network heterogeneity will promote the spread of the software [37]. To the best of our knowledge, consumers' innovation diffusion of decision-making is affected by individual personality and preferences, such as personality traits, decision attitudes and behavior habits, which can be described by agents in the simulation models [38]. Additionally, social networks provide a system that allows users to communicate and interact in the process of innovation diffusion, so that social influence plays an important role in user decision-making [39]. Social influence is often a phenomenon in social networks and may play a critical role in enhancing social interactions between consumers and on innovation behaviors, as well as the opportunities to exploit these interactions [40,41]. For example, agents are aware of the impacts of each connection and decide whether they establish relations with other agents. Therefore, consumers' adoption decisions are influenced by marketing activities as well as positive and negative word-of-mouth communication between neighboring consumers in the social networks [34,42]. In this paper, we explore the influence of social network structures on the decision-making of mobile user groups, investigating the characteristics of innovation diffusion for mobile application under global and local network effects. 


\section{Methodology}

\subsection{Conceptual Design of the Simulation Model}

For the diffusion of mobile application innovation, each user is an independent individual, individuals communicate and interact with each other and then they make their own decisions in the user group. Hereby we design the conceptual model of innovation diffusion in this section. Firstly, we made assumptions about mobile applications in the user group. Secondly, after analyzing the characteristics of user behavior and the influencing factors of decision-making, we constructed the model of user decision-making in innovation diffusion mobile applications. Finally, we described the impact of social networks on the simulation models.

\subsubsection{Mobile Applications Hypothesis}

According to this research $[43,44]$, the premises or assumptions of models are vital in simulating the user decision-making process. Hereby, drawing on those researches, we make the following assumptions about the individual decision-making behavior and initial value of the consumers and the mobile application, modeling the innovation diffusion of mobile applications.

1. Mobile applications are abstractions of common features and attributes of mobile applications that are diffused in the market. The unique features of some mobile applications are not considered, and the changes of functions and characteristics of mobile applications in the diffusion process are always ignored.

2. The diffusion of mobile applications in the market will not be affected by other mobile applications; that is, we only consider the diffusion of one mobile application among the user community.

3. Users will obtain the decision situation and mobile apps utility value from their neighbors (social influence). However, because of their own cognitive and information processing capabilities, they can hardly make long-term predictions based on global information.

4. Users will make different decisions when facing the same application, which is the difference determined by personal preference and personality traits.

\subsubsection{Conceptual Model for Individual Decisions by a Mobile Application User}

Typically, users mainly obtain information about mobile applications in two ways. One is advertisements and information in the public media and application market. The other is social influence, which mainly refers to getting the public's evaluation of mobile applications during user communication and interaction. Users can perceive the existence of mobile applications and their value utility, and make decisions. According to the theory of information processing [45], the individual decision is mainly based on external environmental information and their own preference and decision attitude.

Therefore, we built a conceptual model for individual decisions by a mobile application user. Firstly, users integrate the value utility information of mobile applications, such as perceived benefits, use costs and network effects, as well as information on the neighborhood's decisions, group communication and interaction. Finally, users make the decision of acceptance or rejection according to their personal preferences and personality characteristics.

\subsubsection{Design of the Mobile Application User Decision and Interaction Process}

Based on the conceptual model of mobile application user decision-making and the assumption of the user's individual personality and preference, we designed a user decision-making and interaction process in the diffusion of mobile application innovation as follows. Firstly, we calculated the value of the current decision-making mobile application. We then collected, acquired and stored the state and information of the neighbor's decision. In, addition, user preferences, personality traits and 
communication and interaction with their neighbors will also affect user decisions. By assessing the value of the mobile applications, users can decide to adopt or reject the mobile app at the next moment.

In the process of diffusion of mobile applications, individuals can communicate and interact with other users. Meanwhile, user decision may be affected by neighbors due to social influence. Researchers mainly use game theory to describe the interaction between different individuals [28,29]. Therefore, this paper employs the game matrix to describe the interaction between different users, as shown in Table 1.

Table 1. Game matrix of user decision-making behavior.

\begin{tabular}{ccc}
\hline \multirow{2}{*}{ User 1 } & \multicolumn{2}{c}{ User 2 } \\
\cline { 2 - 3 } & Accept & Refuse \\
\hline Accept & $b-c+m /(n * k) \cdot b-c+m /(n * k)$ & $b-c+m /(n * k) \cdot b-f$ \\
\hline Refuse & $b-f \cdot b-c+m /(n * k)$ & 0,0 \\
\hline
\end{tabular}

In the above table, $b$ represents the utility value of the mobile application; $c$ denotes the use cost of the mobile application; and $f$ is used to express the loss of the benefits due to rejection. We can obtain the return $b-f(b>f)$ when the consumer rejects the product. Furthermore, $n$ represents the total number of user groups, whilst $m$ denotes the marketing and promotion investment from companies. $m /(n * k)$ indicate the value that each user who adopts a mobile app can derive from corporate marketing. Assume that in a fixed time step, the percentage of users who choose to use mobile applications is $k$. Then, we calculate the expected benefits of user adoption in the following equations:

$$
\begin{aligned}
E_{A} & =(b-c+m /(n * k)) * k+(b-c+m /(n * k)) *(1-k) \\
& =b-c+m /(n * k)
\end{aligned}
$$

Meanwhile, the expected benefits of user rejection $E_{B}$

$$
\begin{aligned}
E_{B} & =(b-f) * k+0 *(1-k) \\
& =(b-c) * k
\end{aligned}
$$

Therefore, when someone in the user community adopt the mobile application $k>0$, the revenue from adoption is $b-c+m /(n * k)$, while the revenue from rejection is $(b-f) * k$. If no user adopts this mobile app, the benefit is 0 . In addition, user preferences and network effects also closely relate to the value utility of the mobile applications. Therefore, we calculated the effect of value utility on the individual decision-making of users, which should be composed of three parts: (i) the expected revenue from mobile application, (ii) user preferences, and (iii) the network effect of the mobile application. Then, the value utility of user $i$ make the decision of adopting or rejecting the mobile application at the timestamp $T$.

$$
U_{i}(t)=E_{i}+\operatorname{pre}_{i}+d D_{i}(t-1)
$$

where $E_{i}$ represents the expected benefits of user $i, d$ denotes the network utility of the mobile app, and $\mathrm{pre}_{i}$ refers to the user's preference for the mobile application.

We let $D_{i}(t-1)$ describe the utility value in different networks. $D_{i}(t-1)$ indicates the percentage of adopter in all users under the global networks effect, i.e., the value is $k$ at timestamp $t-1$. Meanwhile, under the local network effect, $N_{i}(t-1)$ represents the number of neighbor users who adopt the mobile application at $t-1$, and we let $z_{i}$ indicate the degree of user $i, D_{i}(t-1)=N_{i}(t-1) / z_{i}$. The utility of the mobile applications to the users is related to the neighbors' decisions in the last timestamp, which can be calculated by the following equation:

$$
U_{a}=b-c+m /(n * k)+\operatorname{pre}_{i}+d * k
$$


Then, we present the value utility function under the local network effect:

$$
U_{a}=b-c+m /(n * k)+\operatorname{pre}_{i}+d * N_{i}(t-1) / z_{i}
$$

However, if users refuse to adopt the mobile application, they cannot enjoy the network externalities of the mobile application. For both types of network effects, we present the value utility function of the user refuse strategy at time $t$ :

$$
U_{b}=(b-f) * k+p r e_{i}
$$

Different from the diffusion in a traditional network, the user in our mobile diffusion system is influenced by individual preferences and neighbors, deciding whether to adopt the mobile application at the next moment. For our bounded rationality, individual users cannot understand the overall situation, whilst the individual can gain knowledge by observing and learning.

In this paper, user preferences and neighbors' decision are all influencing the individual decision-making of mobile applications. Communication and interaction in the social network also impact a consumer's decisions. Hence, we adopted a social learning method to realize the interaction between users. The specific design is shown as follows.

According to the attitude of decision in the mobile application marketing, we described user decision into three types: the conservative, neutral and mutable, which are expressed by $P_{a}, P_{b}$ and $P_{c}$. Each type of user decision threshold followed the probability distributions $P_{a} \sim \operatorname{Uni}(0.7,1)$, $P_{b} \sim$ Uni $(0.4,0.7)$ and $P_{c} \sim U n i(0.4,0)$, respectively.

In addition, users will learn the decision-making from their neighbors, which is related to each other's personality traits. When the decision-making attitudes of the uses are similar, it is more likely to learn from each other. On the contrary, it is difficult to adopt the same decision when individual users want to reflect differences in personality traits from other types of users. In this paper, we present the probability matching method of user decision-making attitude, showing the possibility of decision learning among users.

The probability of matching between individuals is $\operatorname{Match}\left(P_{m}, P_{n}\right) \in(0,1), m, n \in(a, b, c)$. Then, we map the matching probability among the three decision attitudes $\operatorname{Match}\left(P_{1}, P_{1}\right)=\operatorname{Uni}(0.7,1)$, $\operatorname{Match}\left(P_{1}, P_{2}\right)=\operatorname{Uni}(0.4,0.7), \operatorname{Match}\left(P_{1}, P_{3}\right)=\operatorname{Uni}(0,0.4), \operatorname{Match}\left(P_{2}, P_{2}\right)=\operatorname{Uni}(0.7,1)$, $\operatorname{Match}\left(P_{2}, P_{3}\right)=U n i(0.4,0.7), \operatorname{Match}\left(P_{3}, P_{3}\right)=U n i(0.7,1)$.

Drawing on the theory of social influence, users always compare the value utility of the mobile application by obtaining their own decisions and those of the neighboring users. If a user's utility value is higher than all the neighbor users, then he will maintain the current decision state at the next moment without learning from the surroundings. When the utility of the neighbor is higher than that of the user, the user will learn the decision-making strategies of their highest utility neighbors with a certain probability in Equation (7).

$$
P=\frac{1}{1+e^{\frac{-\left(u_{j}-U_{i}\right)}{r}}}
$$

where $U_{i}$ denotes the value utility of the user's own mobile application value, $U_{j}$ indicates the value utility of the imitated object's mobile application value, and $r$ represents the information noise in the process of social learning. When the probability of decision learning exceeds the matching range above, the probability of learning takes the probability of exceeding the decision threshold. Otherwise, we take the current probability value as the decision learning probability. If the user succeeds in imitating someone else's decision, he will maintain his current strategy at the next moment. Then the user may adjust its decision state at a certain probability in the next time step. The user in the social network may constantly interact with each other due to different decision-making strategies and value benefits. 


\subsection{Simulation Model with Multi-Agents}

In this section, the simulation conceptual model will be implemented in Anylogic 8, which is a tool that provide many simulation modeling methods [46]. The simulation model includes the following three parts: (i) the setting of the main model and class; (ii) the steps of model running; and (iii) the setting and verification of model parameters.

\subsubsection{Setting of the Main Model and Class}

According to the simulation conceptual model mentioned in Figure 1, a multi-agent model of mobile application innovation diffusion considering the network effect and network structure was established in Anylogic 8, as shown in Figures 1 and 2.

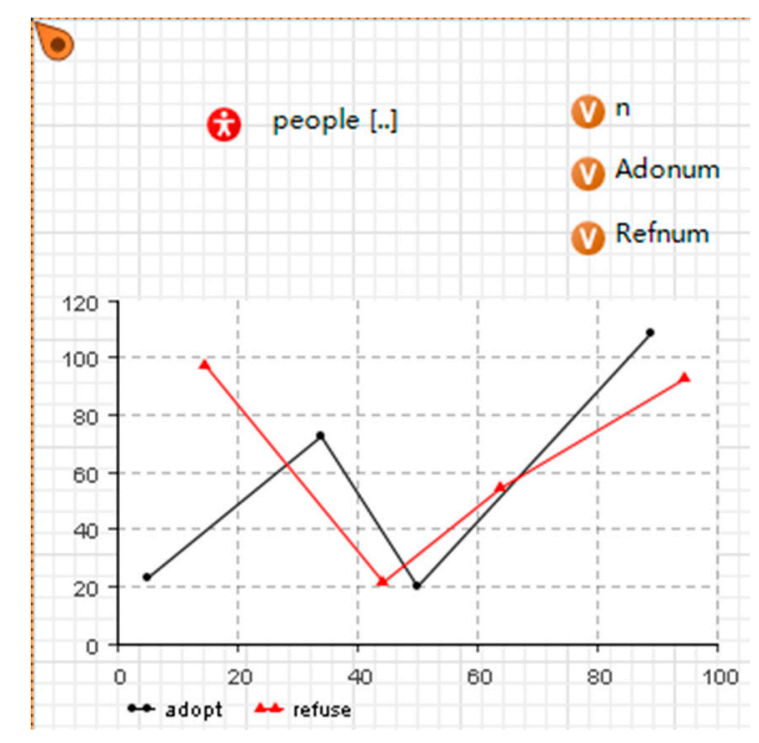

Figure 1. The main multi-agent model.

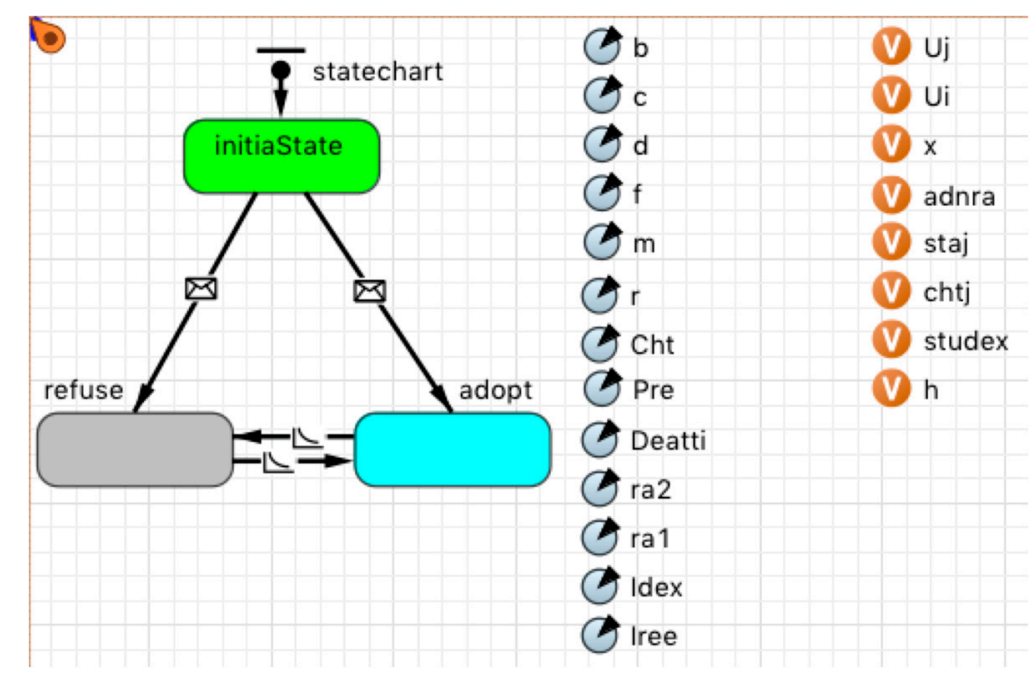

Figure 2. The person category.

Figure 1 describes the main class of the simulation model. The term people refer to the collection of mobile application user agents. Each user is an individual agent. The simulation model supports setting the initial network structure among all user agents in the people class. The network topology can be set directly according to the needs of the simulation experiments. At the initial time of model 
operation, the corresponding network generation function will be called to build the initial networks. Additionally, Adonum indicates the number of users who adopt the mobile application, whilst Refnum represents the number of users who reject the app; $N$ refers to the total number of mobile application users. Then, the bottom chart in Figure 1 shows the change in the number of users who adopt and reject this application.

As shown in Figure 2, each agent is a person object. In addition to the initial state, each agent is divided into two states: adopt and reject. The initial state allows a certain proportion of users as seed users in a random way. The parameters and variables in person are mainly built according to the needs of implementing the user decision-making and interaction process of the mobile applications. The main parameters are presented as follows. Cht denotes personality character and determines the user's decision attitude. $C h t=\{1,2,3\}$ indicates the conservative, balance and mutable, respectively. Pre represents user preferences for mobile applications, which follows the normal distribution $N(u, w)$. In addition, Deatti describes whether the user rejects the mobile app at the timestamp $t$; the parameter type is Boolean. $r a 1$ and $r a 2$ represent the probability of change of the decision state, respectively.adnra denotes the proportion of users who adopt the mobile app in all neighbors, which will be used to calculate the local network effect. Iree represents the user degree value, i.e., the number of neighbors in the social network. Besides, studex describes the learning neighbor agent, staj represents the decision state of the neighbor user and chaj indicts the personality characteristics of the neighbors user.

\subsubsection{Modelling Processes}

According to the conceptual design of simulation model, we first constructed the initial social networks. Then, we realized the process behind user agent decision and interaction in the user community. Finally, we built the multi-agent model of mobile application innovation diffusion with a social network effect. The simulation processes are shown in Table 2.

Table 2. Process of our simulation model.

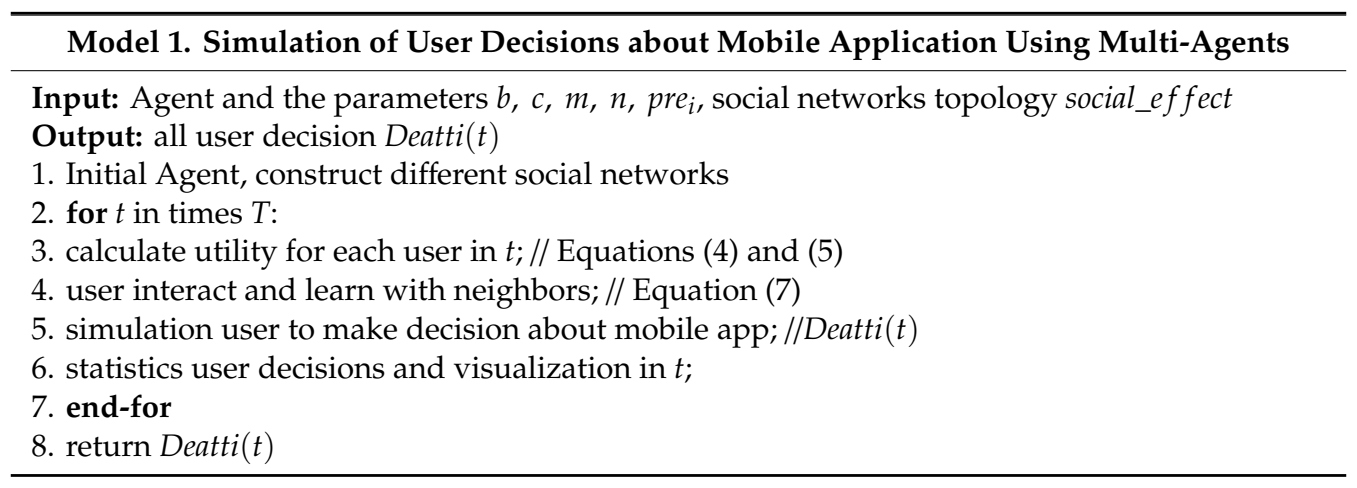

\subsubsection{Model Setting and Validation}

Next, we verified the effectiveness of the simulation model with different individual preferences. According to the research results $[47,48]$, we set the number of initial users as $n=100$; the experimental time was 100 timesteps. Then, the proportion of seed users was $20 \%$; the relevant parameters of the mobile application value utility were $b=100, C=85, f=35, M=0 b=100$. The network effect intensity $D$ was set to 30 . $\phi_{C h t}$ denotes the distributions of user decision preference $C h t$. Then, we employed our simulation model to compare the number of users who adopt or reject the mobile application on different distributions of user personality traits.

In Figure 3a, the number of adopters of mobile apps is growing slowly, because conservative users are less susceptible to other users, maintaining their current decision-making state. Contrasting to Figure 3b, mutable users can easily change their decisions, so there are many spikes in the group decision process. It also suggests that those users are vulnerable to neighbors because of their unstable attitude about the mobile app. In Figure $3 c$, balanced users are more volatile due to their personality 
traits between conservative and mutable. Decision change is significantly lower than that of mutable users. Finally, Figure $3 \mathrm{~d}$ assumes that the distribution of personality characteristics is $30 \%$ conservative, $30 \%$ balanced and $40 \%$ mutable. After the proportion of users adopting mobile applications reaches a certain degree, the change degree of a user group gradually decreases, and basically reaches the stable state of mobile application diffusion in the market.

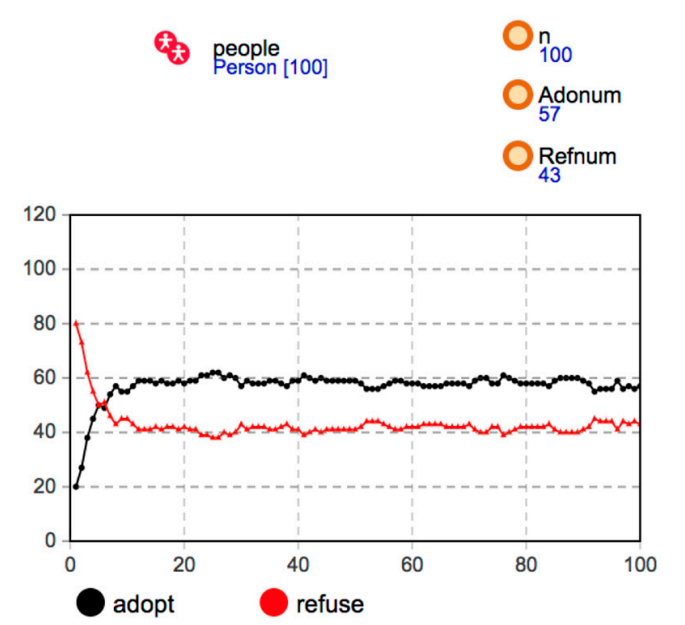

(a)

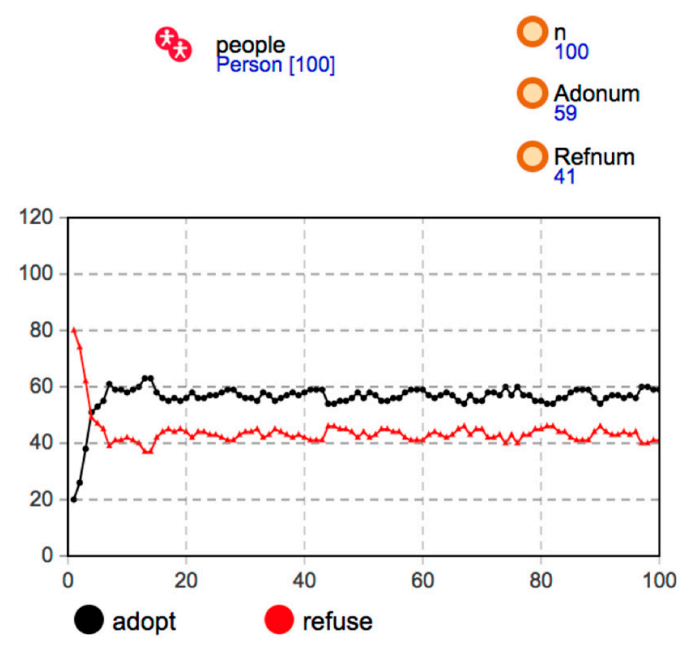

(c)

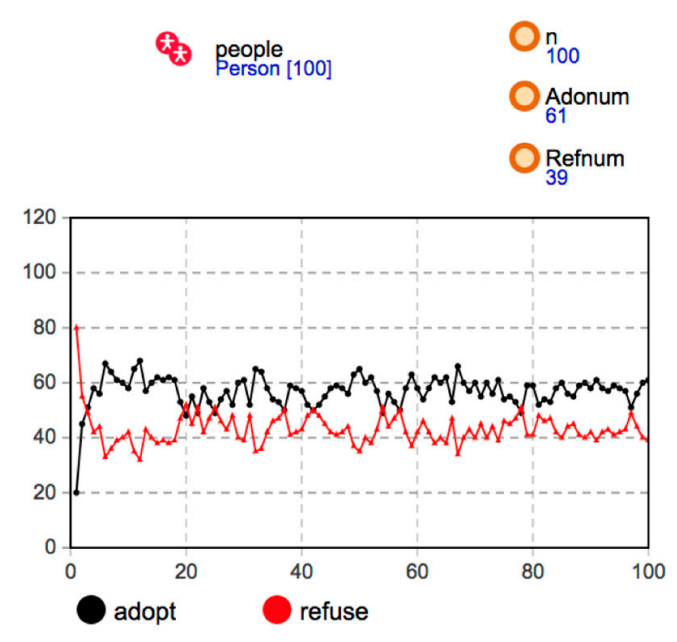

(b)
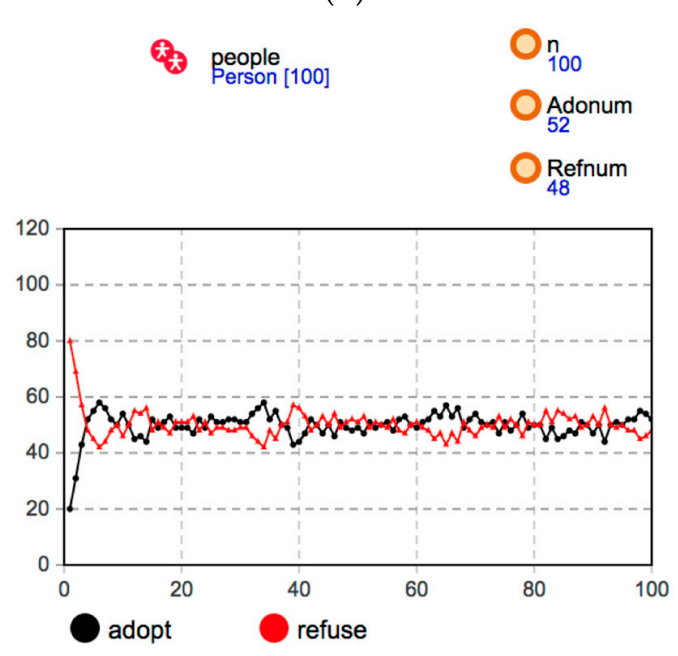

(d)

Figure 3. (a) Distribution of preference $\phi_{C h t}=(1,0,0)$. (b) Distribution of preference $\phi_{C h t}=(0,0,1)$. (c) Distribution of preference $\phi_{C h t}=(0,1,0)$. (d) Distribution of preference $\phi_{\text {Cht }}=(0.3,0.3,0.4)$.

By comparing the simulation result of the above four personality traits with reality, we find that the simulation process and result are consistent with the reality, which shows the simulation model is effective and reliable.

\section{Simulation Results}

In this section, the simulation experiments are explored regarding the internal mechanism and regular characteristics of the innovation diffusion of mobile applications under different network topologies and network effects. More specifically, we first divided the social networks into random networks, small-world networks and scale-free networks. We then investigated the diffusion of the mobile application under different network topologies. Besides, we adjusted the intensity of the network effects to analyze the impact of network effects on innovation diffusion. In this paper, the 
experiments under each environment and parameter were repeated 50 times, and then we took the mean value of the simulation result.

Drawing on the work in [49], we assumed the total number of users was 100 in the simulation experiments. The average degree was 6 . The individual preference was Pre $\sim N(50,10)$, and the user preference distribution always adopts $\phi_{C h t}=(0.3,0.3,0.4)$. We also set $b=120, c=105, f=55, m=0$. The experimental time was 100 timesteps.

\subsection{The Innovative Diffusion of Mobile Applications under Different Network Topology}

We first set the networks type as random networks, small-world networks and scale-free networks, respectively. Then we explored innovative diffusion of mobile applications under different network topologies. The results are shown in Figure 4.

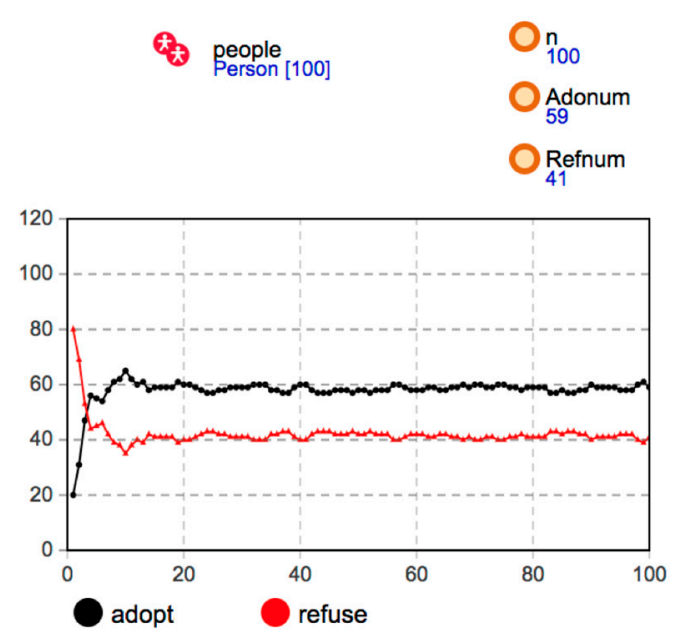

(a)
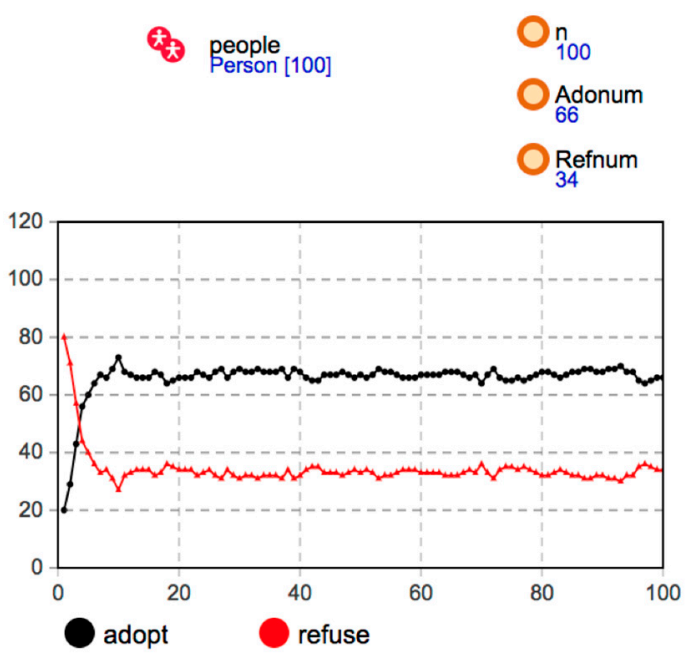

(c)
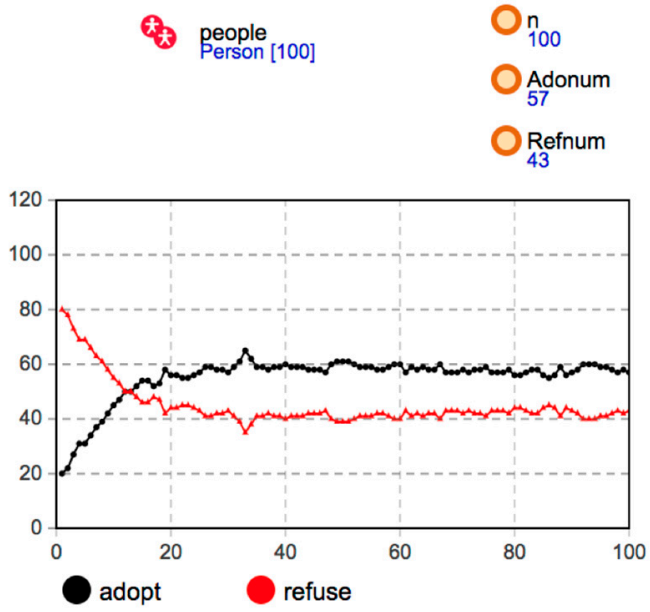

(b)
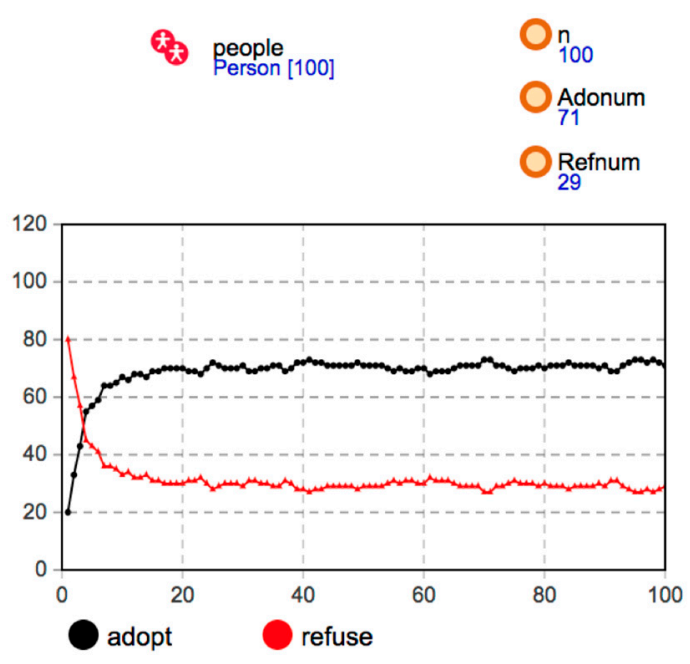

(d)

Figure 4. Cont. 


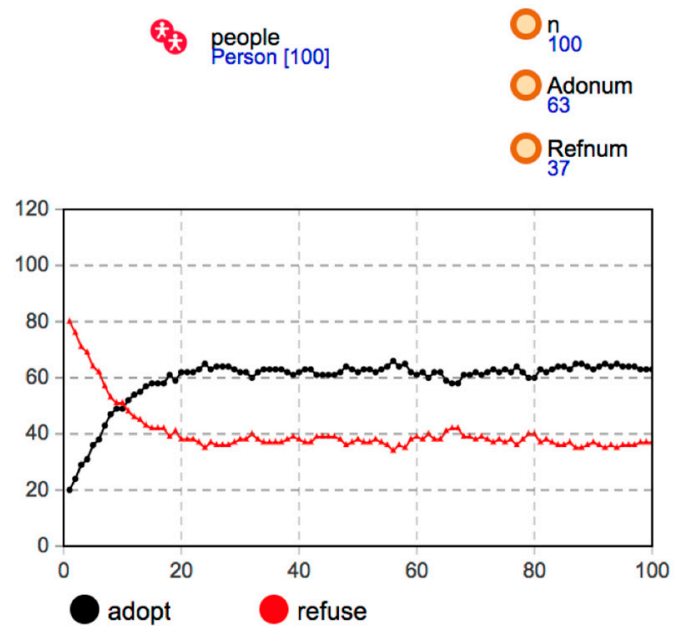

(e)
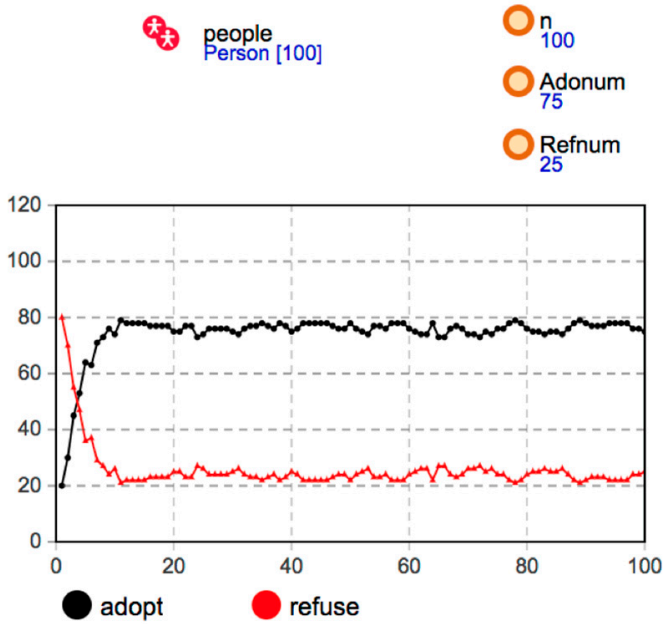

(f)

Figure 4. (a) Random network of global network effect. (b) Small-world network of global network effect. (c) Random network of global network effect. (d) Small-world network of global network effect. (e) Random network of global network effect. (f) Small-world network of global network effect.

\subsubsection{The Diffusion of Mobile Applications under the Global Network Effect}

In Figure 4, disregarding the random network, small-world network and scale-free network, the transmission process of mobile applications has firstly experienced a slow rise and then a decline in the wave process. In terms of diffusion speed, the diffusion speed of random networks and scale-free networks under the global network effect is similar, and group decision begins to stabilize in a relatively short period, and faster than that of the small-world networks. The mobile application can quickly spread in a relatively short time through interaction and social learning behavior. Therefore, the trend of user group decision-making in the three network structures is consistent. The diffusion speed of a mobile application in a random network and scale-free network is similar, whilst in a small-world network it is the slowest and the lowest.

Compared with random networks and small-world networks, there are more users in scale-free networks who finally adopt mobile applications, and the diffusion result of the random networks is slightly better than that of the small-world networks. Although the difference in nodes under the global network effect does not affect the value of the mobile applications, some hub nodes in the scale-free network can obtain the value-utility information of mobile applications. More users also may be willing to communicate with them due to social learning in the user community. After interacting with a large number of neighbors, the behaviors and decisions of the hub nodes may quickly affect their neighbor nodes, promoting the proliferation of mobile applications in the community.

Under the global network effect, there are some high-number nodes in the scale-free network; the communication and interaction between these hub users and the surrounding neighborhood users promote the innovation diffusion of mobile applications, so the diffusion results in the scale-free network is the best.

\subsubsection{The Diffusion of Mobile Applications under the Local Network Effect}

Figure $4 \mathrm{~d}-\mathrm{f}$ present the simulation results under the local network effect. We discover that the evolution trend of the user group decision in three kinds of network structures is similar.

In terms of diffusion results, more users adopt mobile applications under a scale-free network structure, which is also consistent with the global network effect. The difference is that under the local network effect, the disadvantage of a small-world network is more prominent. Since each user in the social network gets the same network value utility under the global network effect, the 
latter is determined by the network effect intensity and the proportion of the number of adopters. However, under the local network effect, the network value utility obtained by the users is related to the decision-making of other users in their neighborhood network. Users in the small world network have a high degree of aggregation. Users in the small group get a similar network effect value, and the decision-making situation is the same. Only a few users will be different from most of the small group due to their preference for mobile applications.

Therefore, compared with the global network effect, mobile application innovation diffusion will be more concentrated in each small group. It is difficult to achieve a better diffusion effect in the whole social network. From the perspective of diffusion speed, the diffusion speed of the scale-free network is the fastest, the random network is slightly lower than the scale-free network, and the diffusion speed of the small-world network is significantly slower than the others. This is because for the mobile application diffusion from one small group to another in a small-world network, which is not conducive to the promotion of diffusion speed, especially in the early stage of diffusion, the diffusion speed is relatively more slowly.

Under the effect of the local network, the diffusion of the three network structures present a similar trend. Due to the relative dispersion of small groups and the difference in the local network effect among groups, the propagation speed and range of the small-world network users are behind the random network and scale-free network, and more obvious than the global network effect.

We also compared the diffusion results of three kinds of network structures under two types of network effects. We then find that more users choose to adopt mobile applications finally under the local network effect, which shows that the local network effect of the same intensity has a more substantial promotion effect on the innovation diffusion of the mobile applications. The impact on user decision-making is more prominent, which is highly consistent with reality. For users, compared with strangers who have no contact among themselves, the neighboring users can often affect their own decision to a greater extent. Under the local network effect, the scale-free network height nodes can not only interact with the surrounding neighbors, but also affect the value utility of mobile applications to users and their decision-making. At the same time, the local network effect further enlarges the differences in a user's product preferences, personality characteristics and neighborhood network, and the uncertainty of a user's decision is also increased under social learning behavior. The fluctuation degree of the user group's decision-making evolution is more obvious than other networks. Therefore, we conclude the following: Compared with the global network effect, the local network effect has a better role in promoting the innovation diffusion of mobile applications. Under the local network effect, the high number of nodes in the scale-free network has a more influential role in promoting the diffusion of mobile application innovation. The differences in user preferences, personality characteristics and neighbor networks are further amplified, and the volatility of user group decision-making evolution is higher under social learning behavior.

\subsection{The Innovative Diffusion of Mobile Applications under a Network Effect}

We adjusted the intensity of the network effect to conduct sensitivity analysis experiments and explored the impact of network effect intensity on innovation diffusion of mobile applications. According to above conclusion, the heterogeneity of the scale-free network degree distribution better reflects the situation in real society. Therefore, the scale-free network is used in the network structure of this experiment. The relevant parameters in the model are set as follows: $B=130, C=110, f=55$, $M=0$. The simulation results are shown in Figure 5. 


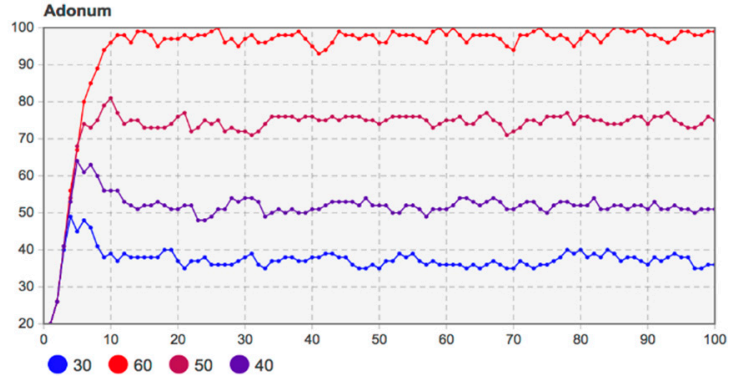

(a)

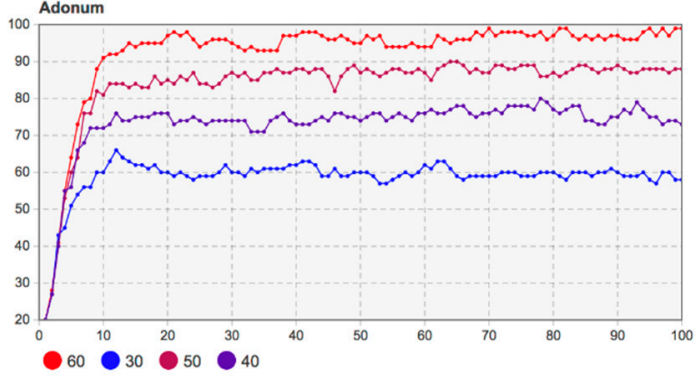

(b)

Figure 5. (a) Sensitivity analysis of network effect intensity under the global network effect $(d=30,40$, $50,60)$. (b) Sensitivity analysis of network effect intensity under the local network effect $(d=30,40$, $50,60)$.

From the simulation results in Figure 5, we find that, with the increase in network effect intensity, mobile applications are further spread in the user community; mobile application propagation is also improved. With the increase in network effect intensity and the value utility of the mobile applications, users who initially refused mobile applications may change their decisions due to social influence. Under the influence of social learning, the number of adopters gradually increases, which is similar to the snowball effect. However, in the case of a low intensity network effect, the value of the network effect cannot change obviously with the increase in the number of users, and communication is difficult to increase in the original degree. Disregarding the local network effect or the global network effect, we discover that the intensity of the network effect can improve the value utility of mobile applications and extend the duration of innovation diffusion. In the diffusion process, the number of users and the value of the network effect promote each other, and finally get better diffusion results.

In addition, comparing the results under the two network effects, it can be found that the diffusion results under the local network effect are often better under the same network effect intensity. Under the local network effect, the increase in network effect intensity can promote the diffusion speed of mobile application innovation diffusion in the early stage, while the diffusion speed under the global network effect will not be affected by the network effect intensity.

\subsection{The Innovative Diffusion of Mobile Applications under Firm Promotion}

We adjusted the firm promotion to conduct sensitivity analysis of marketing experiments, in which we set $m$ as 10, 20 and 30, respectively. The rest of the parameters in the model were $B=130, C=120, f=55$. We then set the proportion of seed users as $10 \%$. The simulation results are shown in Figure 6.

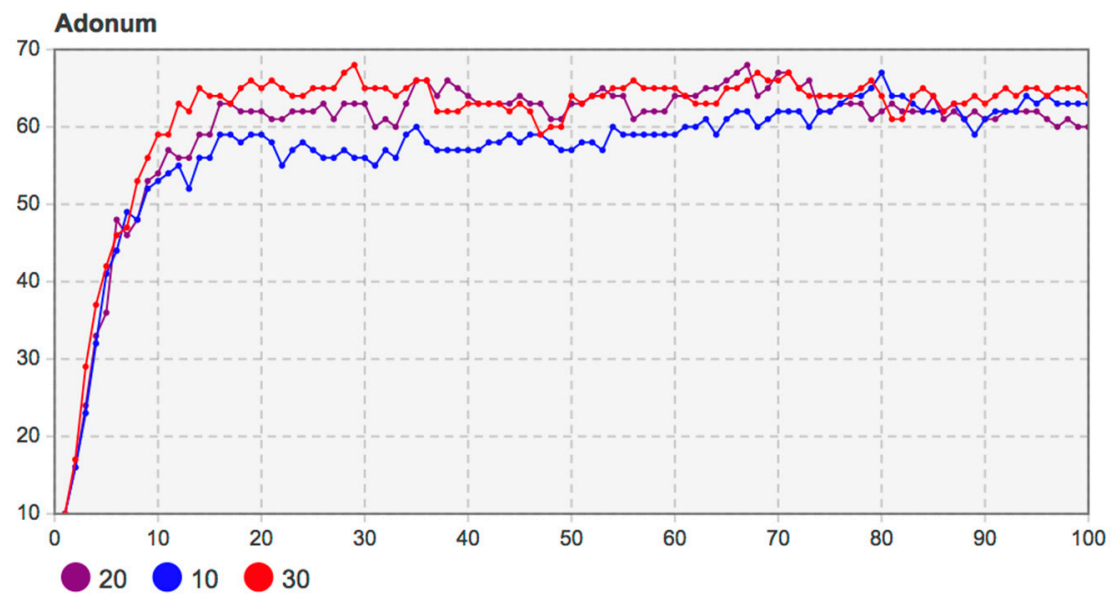

Figure 6. Sensitivity analysis of marketing promotion input to the number of adopters $(m=10,20,30)$. 
In Figure 6, with the increase in marketing promotion investment, the number of users adopting mobile applications has increased in the early diffusion. However, since the time of $T=30$, the advantages of high marketing and promotion investment have gradually decreased. Mobile applications with less marketing and promotion investment have gradually spread. The number of adopters has also gradually increased, and the promotion effect of marketing and promotion investment on diffusion is not obvious.

At the end of diffusion, the gap between the number of adopters under three different marketing inputs is much smaller than that at the beginning, because the number of adopters is smaller than that at the beginning of diffusion. Besides, on the premise of marketing promotion investment, the value income of each adopter is reduced. At the same time, under the evolution of social learning behavior and social network, user decision-making is more affected by the network effect and interactive communication, and the role of marketing and promotion investment is weakening. Therefore, the diffusion trend under different marketing and promotion investment tends to be the same in the later stage, with little difference in diffusion degree.

An enterprise's investment in marketing promotion, which helps promote mobile applications in the initial stage of market entry, has a positive effect on the speed and scale of initial diffusion, but the co-evolution of social network structure and user group decision-making makes this kind of promotion have a relatively weak role in the late period of diffusion; it is difficult to show obvious differences in diffusion results.

\section{Conclusions}

In this paper, we developed and implemented a simulation model based on agent-based modelling, game theory and social networks, to simulate the innovation diffusion of mobile applications in user groups. Firstly, we built a simulation model to describe the individual decision-making process under user preferences and neighbors' decisions in social networks. Then, we built multi-agent models of mobile application innovation diffusion that considers network effects and network structures. Finally, we investigated the impact of firm promotion on diffusion through simulation experiments, which help enterprises make better strategies.

We found that both in the local networks effect and global networks effect, user groups have similar diffusion trends under different social networks structures. Then, the intensity of the network effect can improve the value utility of the mobile applications and extend the duration of innovation diffusion. In line with the research of Wei et al. [3] and Muller and Peres [7], this research evidence also supports the important role of network structure in the diffusion of mobile application innovation. Our findings also emphasize the impact of the user's preferences and neighbor's influence on personal decisions. Several prior studies have examined the relationship between user preferences and decision-making based on multi-agent models [50,51]. They also seek to take in the advantage of this granularity in order to explore the impact of user preferences on the modal split of shared autonomous vehicles in the area of transport. In addition, firm investment in a marketing promotion, which helps to promote mobile applications in the initial stage of market entry, has a positive effect on the speed and scale of the initial diffusion.

Our findings provide theoretical exploration and policy decision support for firms to simulate user behaviors in diffusion of mobile applications. Our research suggests firms should pay attention to individual personalities and neighbor customers' decision information in user groups. Our research can also simulate firm strategies about mobile applications before applying them in reality, which could help enterprises make better strategies and enhance the performance for innovation diffusion of mobile applications.

However, some limitations in the discussion also exist. We have not yet considered the complexity of competition among different mobile applications. In addition, as in many field of multi-agent models, only empirical data are verified in agent-based models with different social networks [34,52]. Although the empirical analysis has not been carried out, we will employ the real data and conclusions 
collected from empirical research to confirm the rationality of the parameter setting in future research. Then we will promote the model to effectively predict mobile application diffusion and develop better business strategies.

Author Contributions: L.Z.: Investigation; software; validation; writing—review and editing; J.L.: Supervision; project administration; Y.L.: Data curation; formal analysis; visualization; writing-original draft; Z.Z: Methodology; resources; writing-original draft; funding acquisition. All authors have read and agreed to the published version of the manuscript.

Funding: This research is supported by the grants of the National Natural Science Foundation of China (No. 71672128).

Acknowledgments: The authors would like to thank the editor and anonymous reviewers for helpful comments and suggestions, which helped to improve the manuscript.

Conflicts of Interest: The authors declare no conflict of interest.

\section{References}

1. Schlüter, M.; Baeza, A.; Dressler, G.; Frank, K.; Groeneveld, J.; Jager, W.; Janssen, M.A.; McAllister, R.R.J.; Müller, B.; Orach, K.; et al. A framework for mapping and comparing behavioral theories in models of social-ecological systems. Ecol. Econ. 2017, 131, 21-35. [CrossRef]

2. Soheila, B.; Atkin, B.; Landin, A. Innovation diffusion through standardization: A study of building ventilation products. J. Eng. Technol. Manag. 2019, 54, 56-66.

3. Wei, X.; Chen, W. How does a firm's previous social network position affect innovation? Evidence from chinese listed companies. Sustainability 2019, 11, 1191. [CrossRef]

4. Amini, M.; Wakolbinger, T.; Racer, M.; Nejad, M.G. Alternative supply chain production-sales policies for new product diffusion: An agent-based modeling and simulation approach. Eur. J. Oper. Res. 2012, 216, 301-311. [CrossRef]

5. Rai, V.; Henry, A.D. Agent-based modelling of consumer energy choices. Nat. Clim. Chang. 2016, 6, 556-562. [CrossRef]

6. Zhang, H.; Vorobeychik, Y. Empirically grounded agent-based models of innovation diffusion: A critical review. Artif. Intell. Rev. 2019, 52, 707-741. [CrossRef]

7. Muller, E.; Peres, R. The effect of social networks structure on innovation performance: A review and directions for research. Int. J. Res. Mark. 2019, 36, 3-19. [CrossRef]

8. Kim, M.J.; Lee, C.K.; Contractor, N.S. Seniors' usage of mobile social network sites: Applying theories of innovation diffusion and uses and gratifications. Comput. Hum. Behav. 2019, 90, 60-73. [CrossRef]

9. Shi, X.; Chumnumpan, P. Modelling market dynamics of multi-brand and multi-generational products. Eur. J. Oper. Res. 2019, 279, 199-210. [CrossRef]

10. Song, P.; Xue, L.; Rai, A.; Zhang, C. The ecosystem of software platform: A study of asymmetric cross-side network effects and platform governance. Mis Q. 2018, 42, 121-142. [CrossRef]

11. Gibbons, D.E. Network structure and innovation ambiguity effects on diffusion in dynamic organizational fields. Acad. Manag. J. 2004, 47, 938-951.

12. Tseng, F.C.; Pham, T.L.; Cheng, T.C.E.; Teng, C.I. Enhancing customer loyalty to mobile instant messaging: Perspectives of network effect and self-determination theories. Telemat. Inform. 2018, 35, 1133-1143. [CrossRef]

13. Fu, W.; Wang, Q.; Zhao, $X$. The influence of platform service innovation on value co-creation activities and the network effect. J. Serv. Manag. 2017. [CrossRef]

14. Jiang, G.; Shang, J.; Liu, W.; Feng, X.; Lei, J. Modeling the dynamics of online review life cycle: Role of social and economic moderations. Eur. J. Oper. Res. 2020, in press. [CrossRef]

15. Yi, H.; Berry, F.S.; Chen, W. Management innovation and policy diffusion through leadership transfer networks: An agent network diffusion model. J. Public Adm. Res. Theory 2018, 28, 457-474. [CrossRef]

16. Bass, F.M. Comments on a new product growth for model consumer durables the bass model. Manag. Sci. 2004, 50, 1833-1840. [CrossRef]

17. Ntwoku, H.; Negash, S.; Meso, P. CT adoption in Cameroon SME: Application of Bass diffusion model. Inf. Technol. Dev. 2018, 23, 296-317. [CrossRef] 
18. Rao, K.U.; Kishore, V.V.N. A review of technology diffusion models with special reference to renewable energy technologies. Renew. Sustain. Energy Rev. 2010, 14, 1070-1078. [CrossRef]

19. North, M.J.; Macal, C.M.; Aubin, J.S.; Thimmapuram, P.; Bragen, M.; Hahn, J.; Karr, J.; Brigham, N.; Lacy, M.E.; Hampton, D. Multiscale agent-based consumer market modeling. Complexity 2010, 15, 37-47. [CrossRef]

20. Garcia, R.; Jager, W. From the special issue editors: Agent-based modeling of innovation diffusion. J. Prod. Innov. Manag. 2011, 28, 148. [CrossRef]

21. Macal, C.M. Everything you need to know about agent-based modelling and simulation. J. Simul. 2016, 10, 144-156. [CrossRef]

22. Kiesling, E.; Günther, M.; Stummer, C.; Wakolbinger, L.M. Agent-based simulation of innovation diffusion: A review. Eur. J. Oper. Res. 2012, 20, 183-230. [CrossRef]

23. Jiang, G.; Tadikamalla, P.R.; Shang, J.; Zha, L. Impacts of knowledge on online brand success: An agent-based model for online market share enhancement. Eur. J. Oper. Res. 2016, 248, 1093-1103. [CrossRef]

24. Ma, T.; Nakamori, Y. Agent-based modeling on technological innovation as an evolutionary process. Eur. J. Oper. Res. 2005, 166, 741-755. [CrossRef]

25. Stummer, C.; Kiesling, E.; Günther, M.; Vetschera, R. Innovation diffusion of repeat purchase products in a competitive market: An agent-based simulation approach. Eur. J. Oper. Res. 2015, 245, 157-167. [CrossRef]

26. Gibbons, R.S. Game Theory for Applied Economists; Princeton University Press: Princeton, NJ, USA, 1992.

27. Abapour, S.; Nazari-Heris, M.; Mohammadi-Ivatloo, B.; Hagh, M.T. Game theory approaches for the solution of power system problems: A comprehensive review. Arch. Comput. Methods Eng. 2020, 27, 81-103. [CrossRef]

28. Ozkan-Canbolat, E.; Beraha, A.; Bas, A. Application of evolutionary game theory to strategic innovation. Procedia Soc. Behav. Sci. 2016, 235, 685-693. [CrossRef]

29. Zhang, R.; Sun, B. A competitive dynamics perspective on evolutionary game theory, agent-based modeling, and innovation in high-tech firms. Manag. Decis. 2019. [CrossRef]

30. Ji, P.; Ma, X.; Li, G. Developing green purchasing relationships for the manufacturing industry: An evolutionary game theory perspective. Int. J. Prod. Econ. 2015, 166, 155-162. [CrossRef]

31. Rahmandad, H.; Sterman, J. Heterogeneity and network structure in the dynamics of diffusion: Comparing agent-based and differential equation models. Manag. Sci. 2008, 54, 998-1014. [CrossRef]

32. Kim, H.; Park, Y. Structural effects of R\&D collaboration network on knowledge diffusion performance. Expert Syst. Appl. 2009, 36, 8986-8992.

33. Luo, S.; Du, Y.; Liu, P.; Xuan, Z.; Wang, Y. A study on coevolutionary dynamics of knowledge diffusion and social network structure. Expert Syst. Appl. 2015, 42, 3619-3633. [CrossRef]

34. Will, M.; Groeneveld, J.; Frank, K.; Müller, B. Combining social network analysis and agent-based modelling to explore dynamics of human interaction: A review. Socio-Environ. Syst. Model. 2020, 2, 16325. [CrossRef]

35. Liu, W.; Wu, X.; Yang, W.; Zhu, X.; Zhong, S. Modeling cyber rumor spreading over mobile social networks: A compartment approach. Appl. Math. Comput. 2019, 343, 214-229. [CrossRef]

36. Mallory, A.; Crapper, M.; Holm, R.H. Agent-Based Modelling for Simulation-Based Design of Sustainable Faecal Sludge Management Systems. Int. J. Environ. Res. Public Health 2019, 16, 1125. [CrossRef] [PubMed]

37. Choi, H.; Lee, B. Examining network externalities and network structure for new product introduction. Inf. Technol. Manag. 2012, 13, 183-199. [CrossRef]

38. Delre, S.A.; Jager, W.; Bijmolt, T.H.A.; Janssen, M.A. Will it spread or not? The effects of social influences and network topology on innovation diffusion. J. Prod. Innov. Manag. 2010, 27, 267-282. [CrossRef]

39. Palos-Sanchez, P.R.; Correia, M.B.; Saura, J.R. An empirical examination of adoption of mobile applications in Spain and Portugal, based in UTAUT. Int. J. Mob. Commun. 2019, 17, 579-603. [CrossRef]

40. Blascovich, J. Social Influence within Immersive Virtual Environments; The social life of avatars; Springer: London, UK, 2002; pp. 127-145.

41. Fox, J.; Ahn, S.J.; Janssen, J.H.; Yeykelis, L.; Segovia, K.Y.; Bailenson, J.N. Avatars versus agents: A meta-analysis quantifying the effect of agency on social influence. Hum. Comput. Interact. 2015, 30, 401-432. [CrossRef]

42. Veglis, A.; Maniou, T.A. The Mediated Data Model of Communication Flow: Big Data and Data Journalism. Kome Int. J. Pure Commun. Inq. 2018, 6, 32-43. [CrossRef]

43. Bryson, J.J.; Ando, Y.; Lehmann, H. Agent-based modelling as scientific method: A case study analysing primate social behaviour. Philos. Trans. R. Soc. B Biol. Sci. 2007, 362, 1685-1699. [CrossRef] [PubMed] 
44. Joseph, J.; Gaba, V. Organizational Structure, Information Processing, and Decision-Making: A Retrospective and Road Map for Research. Acad. Manag. Ann. 2020, 14, 267-302. [CrossRef]

45. Hann, I.H.; Hui, K.L.; Lee, S.Y.T.; Png, I.P.L. Overcoming online information privacy concerns: An information-processing theory approach. J. Manag. Inf. Syst. 2007, 24, 13-42. [CrossRef]

46. Nikolai, C.; Madey, G. Tools of the trade: A survey of various agent-based modeling platforms. J. Artif. Soc. Soc. Simul. 2009, 12, 2.

47. Carlos, E.; Oteiza-Aguirre, N. An agent based multi-optional model for the diffusion of innovations. Phys. A Stat. Mech. Appl. 2014, 394, 254-265.

48. Yu, X.; Han, J. Forecasting new product diffusion with agent-based models. Technol. Forecast. Soc. Chang. 2016, 105, 167-178.

49. Verhoef, P.C.; Stephen, A.T.; Kannan, P.K.; Luo, X.; Abhishek, V.; Andrews, M.; Bart, Y.; Datta, H.; Fong, N.; Hoffman, D.L.; et al. Consumer connectivity in a complex, technology-enabled, and mobile-oriented world with smart products. J. Interact. Mark. 2017, 40,1-8. [CrossRef]

50. Kickhöfer, B.; Grether, D.; Nagel, K. Income-contingent user preferences in policy evaluation: Application and discussion based on multi-agent transport simulations. Transportation 2011, 38, 849. [CrossRef]

51. Kamel, J.; Vosooghi, R.; Puchinger, J.; Ksontini, F.; Sirin, G. Exploring the impact of user preferences on shared autonomous vehicle modal split: A multi-agent simulation approach. Transp. Res. Procedia 2019, 37, 115-122. [CrossRef]

52. Laatabi, A.; Marilleau, N.; Nguyen-Huu, T.; Hbid, H.; Babram, M.A. ODD+ 2D: An ODD based protocol for mapping data to empirical ABMs. J. Artif. Soc. Soc. Simul. 2018, 21, 9. [CrossRef]

(C) 2020 by the authors. Licensee MDPI, Basel, Switzerland. This article is an open access article distributed under the terms and conditions of the Creative Commons Attribution (CC BY) license (http://creativecommons.org/licenses/by/4.0/). 